\title{
Scrub Typhus: An Uncommon Cause of Pyrexia without Focus
}

\author{
Yadav D', Chopra A², Dutta AK ${ }^{3}$, Kumar S $^{4}$, \\ Kumar $\mathbf{V}^{5}$
}

${ }^{1}$ Dr. Dinesh Yadav, MBBS, MD, ${ }^{2}$ Dr. Abhishek Chopra, MBBS, MD, ${ }^{3}$ Dr. Ashok Kumar Dutta, MD, FIAP, ${ }^{4}$ Dr. Sandeep Kumar, MBBS, MD, ${ }^{5}$ Dr. Virendra Kumar, MBBS. MD. All from the Paediatric Intensive Care Unit, Department of Paediatrics, Lady Hardinge Medical College and associated Kalawati Sharan Children's Hospital, New Delhi-110001.

\section{Introduction}

crub typhus is a type of rickettsial disease caused by the bite of an infected larval mite or "chiggers" belonging to the family Trombiculidae, genus and subgenus Leptotrombidium. The agent responsible for Scrub typhus is Orientia tsutsugamushi ${ }^{1}$. Commonly reported from hilly and forest areas, it has not been reported from Delhi or other urban areas. We hereby report a child resident of Delhi, who presented with pyrexia without focus and was subsequently diagnosed as scrub typhus.

\section{Case}

A seven year male child resident of New Delhi presented with high grade (up to $105^{\circ} \mathrm{F}$ ) continuous fever with chills and rigors for eight days. Child also had history of cough and vomiting for 5 days. He had small transient erythematous macular rash over the chest at beginning of fever, which subsided by next day spontaneously without residual pigmentation. There was no history of loose stools, respiratory distress, dysuria, bleeding from any site, jaundice, alteration in sensorium or recent visit outside Delhi. At admission, child was hemodynamically stable. There was no pallor, icterus, bleeding spots or significant lymphadenopathy. Physical examination did not reveal any evidence of eschar, rash or organomegaly. Respiratory, cardiovascular and nervous system examination were within normal limits.

His initial blood investigations revealed leukocyte count $12,200 / \mathrm{mm}^{3}$ with neutrophilia with shift to left and

\section{Address for correspondence \\ Dr. Dinesh Yadav \\ E-mail: dineshmamc@gmail.com}

This work is licensed under a Creative Commons Attribution 3.0 License.

\begin{abstract}
Scrub typhus is a rickettsial disease caused by Orientia tsutsugamushi. Commonly reported from hilly and forest areas, it usually presents with fever, eschar, maculopapular rash, headache, gastrointestinal symptoms and lymphadenopathy. We report a seven year old male resident of Delhi, a non-endemic region, presenting with pyrexia without focus and fluid refractory hypotension, subsequently diagnosed as scrub typhus. Fever responded to doxycycline and child was discharged on day 5. To conclude, rickettsial infections can be seen in non-endemic, urban areas as well and should be suspected and investigated in children presenting with pyrexia without focus, not responding to usual antibiotics.
\end{abstract}

Key words: Scrub typhus, Orientia tsutsugamushi.

toxic granules in neutrophils with normal hemoglobin and platelet count. Renal, liver function tests and serum electrolytes were within normal limits. Peripheral smear, rapid malaria antigen test, widal and typhidot lgM were negative. Blood and urine cultures were sterile. Chest X-ray was normal and tuberculin test was negative. Ultrasonography abdomen revealed normal size of liver and spleen with multiple mesenteric lymph nodes (largest $13.6 \times 10.6 \mathrm{~mm})$. Parenteral ceftriaxone was started considering possibility of enteric fever. However, the child continued to have high grade fever and on $3^{\text {rd }}$ day of admission, developed tachycardia and hypotension, which was managed with fluid boluses and ionotropes. Antibiotics were upgraded to meropenem and linezolid. Shock responded to vasopressors; however, fever persisted despite 48 hours of $2^{\text {nd }}$ line antibiotics. Further investigations for pyrexia without focus revealed negative rheumatoid factor, antinuclear antibody, and leptospira and dengue serology. Echocardiography did not reveal any evidence of infective endocarditis. Weil-Felix test revealed increased OX K antibody titres (1:160) with normal titres of OX 2 and OX 19, suggestive of scrub typhus. Child was started on oral doxycycline. Subsequently, positive lgM antibody test for Orientia

How to cite this article?

Yadav D, Chopra A, Dutta AK, Kumar S, Kumar V. Scrub Typhus: An Uncommon Cause of Pyrexia without Focus. J Nepal Paediatr Soc 2013;33(3):234-235. 
tsutsugamushi confirmed the diagnosis of scrub typhus. Defervescence occurred within 48 hours and child was discharged on day 5 of doxycycline. Retrospective interrogation regarding source of infection revealed that child used to play in nearby park surrounded by lot of bushes, which might have been the potential source of mite. Macular rash prior to admission might have been part of scrub typhus spectrum; however, absence of eschar and other characteristic features of typhus infection delayed the suspicion of diagnosis.

\section{Discussion}

Scrub typhus is a rickettsial disease caused by Orientia tsutsugamushi and spread by bite of the larvae of Trombiculid deliniensis mite in India. It occurs in a geographical area known as "tsutsugamushi triangle" which extends from northern Japan to northern Australia and Pakistan and Afghanistan ${ }^{2}$. Scrub typhus is endemic in India and has commonly been reported from hilly and forest areas $^{3}$, however, it has not been reported from urban areas.

Eschar and maculopapular rash are most characteristic features of scrub typhus, when present. However, eschar is rare in Southeast Asian patients and was reported in $5.8 \%$ cases from himalayan region ${ }^{4}$. Other symptoms and signs include fever with chills, headache, cough, gastrointestinal symptoms, lymphadenopathy and hepatomegaly ${ }^{5}$. Complications include meningoencephalitis, interstitial pneumonia, myocarditis, disseminated intravascular coagulation and multiorgan failure ${ }^{6}$.

Our patient did not have any of the characteristic features of scrub typhus, nor did he belong to an endemic region. Scrub typhus presenting as pyrexia without focus have been reported in $7.5 \%$ cases from Thailand ${ }^{7}$ and in $15 \%$ cases in a recent case series from South India ${ }^{8}$. However, no nationwide data is available from India on the magnitude of the problem and exact contribution of scrub typhus to cases with pyrexia without focus from non endemic regions is not reported. Fluid refractory hypotension was another unusual feature in present case. Delayed administration of doxycycline was reported to be associated with major organ dysfunction including myocarditis, hypotension and prolonged ICU stay ${ }^{9}$.

The mainstay in scrub-typhus diagnostics remains serology. The oldest test in current use is the Weil-Felix OX-K agglutination reaction, which is inexpensive, easy to perform, and results are available overnight; however, it lacks specificity and sensitivity. The indirect fluorescent antibody (IFA) test is more sensitive, and results are available in a couple of hours; however, the test is more expensive and requires considerable training. IFA uses fluorescent anti-human antibody to detect specific antibody from patient serum bound to a smear of scrubtyphus antigen and is currently the reference standard ${ }^{10}$.

Doxycycline, tetracycline and chloramphenicol are the mainstay of treatment. Treatment duration extends for 5-7 days or till 3 days of minimum afebrile period ${ }^{11}$. Our patient was treated with doxycycline and responded well.

To conclude, rickettsial infections can be seen in non-endemic, urban areas as well in present era of globalization, as in the present case. Hence, these infections should be suspected and investigated in any child presenting with pyrexia without focus, not responding to usual antibiotics, even in the absence of characteristic features of the disease.

\section{References}

1. Digra SK, Saini GS, Singh V, et al. Scrub typhus in children: Jammu experience. JK Science 2010;2(12):95-7.

2. Chogle AR. Diagnosis and treatment of scrub typhus- the Indian scenario. J Assoc Physicians India 2010;58:11-2.

3. Mahajan SK, Kashyap R, Kanga A, et al. Relevance of Weil-Felix test in diagnosis of scrub typhus in India. J Assoc Physicians India 2006;54:619-21.

4. Mahajan SK, Rolain JM, Kashyap R, et al. Scrub typhus in Himalayas. Emerg Infect Dis 2006;12(10):1590-2.

5. Sin NC, Yau FT, Luk WK. A case of scrub typhus in child. HK J Pediatr 2000;5:45-7.

6. Kulkarni A. Childhood rickettsiosis. Ind $J$ Pediatr 2011;78:81-7.

7. Sirisanthana V, Puthanakit T, Sirisanthana T. Epidemiology, clinical and laboratory features of scrub typhus in thirty Thai children. Pediatr Infect Dis J 2003;22:341-5.

8. Somashekhar HR, Moses PD, Pavithran S, et al. Magnitude and features of Scrub typhus and Spotted fever in children in India. J Trop Pediatr 2005;52(3):228-9.

9. Lee N, Margaret Ip, Wong B, et al. Risk Factors Associated with Life-threatening Rickettsial Infections. Am J Trop Med Hyg 2008; 78(6): 973978.

10. Koh GC, Maude RJ, Paris DH, Newton PN, Blacksell SD. Diagnosis of scrub typhus. Am J Trop Med Hyg 2010; 82(3):368-70.

11. Liu Q, Panpanich R. Antibiotics for treating scrub typhus (Review). The Cochrane Library 2010, Issue 7. Art. No: CD002150. DOI: 10.1002/14651858. CD002150. 